\section{Specialization of the cerebral hemispheres in scanning for information}

$$
\text { in short-term memory* }
$$

\author{
ROBERTA L. KLATZKY and RICHARD C. ATKINSON \\ Stanford University, Stanford, California 94305
}

Lateral specialization of function in the cerebral hemispheres was investigated in the context of a memory scanning paradigm. The $S$ first memorized a set of letters (the memory set) and then indicated whether or not a subsequent test stimulus, presented in either the right or left visual field, matched any letter in the set. The test stimulus was either a letter or a picture of some common object; for the picture, S's response was based on the initial letter of the name of that object. Reaction time was recorded and plotted as a function of the number of letters in the memory set. The results support the hypothesis that in a memory scanning task of this type, letter and picture test stimuli are spatially and verbally represented, respectively, and are processed in different cerebral hemispheres.

Research on human patients with surgically separated cerebral hemispheres indicates that the left and right hemispheres differ with respect to their modes of information processing. The left hemisphere appears to perform analytic functions, whereas the right hemisphere specializes in Gestalt, holistic processing. Accordingly, a left-hemisphere superiority for the processing of verbal information has long been recognized, and more recently, evidence has been found for right-hemisphere superiority in processing spatial or pattern information (Levy, 1969; Levy-Agresti \& Sperry, 1968; Sperry \& Levy, 1970). In cerebrally intact $S s$, the differences in processing may be revealed with a reaction-time measure (Filbey \& Gazzaniga, 1969). When information presented to one hemisphere must be processed by the other, a reliable increment in response latency is obtained, an increase which may be associated with transfer of the incoming information to the appropriate hemisphere.

In this experiment, lateral specialization of brain function was investigated in the context of a memory scanning paradigm. One form of this task consists of a series of trials of the following nature. On each trial, a set of letters (the memory set) is presented to $S$ to be maintained in short-term memory; the size of this set varies from trial to trial. Then $S$ is presented with a single letter (the test stimulus) and required to indicate manually whether or not that letter matches one of the members of the memory set (a positive or negative response, respectively). The S's

*We wish to thank Dr. C. R. Hamilton for his helpful suggestions. reaction time, defined as the interval between the test stimulus onset and the response, is recorded. Of particular interest in this task are the functions which relate reaction time to memory-set size. These reaction-time functions are consistently linear and increasing. Moreover, the functions for positive and negative responses have approximately the same slope over a wide range of scanning tasks (Sternberg, 1969).

A theory of memory scanning proposed by Sternberg (1966) divides the scanning process into four stages. During Stage 1, the test stimulus is processed and transformed into a representation comparable to the memory-set elements. During Stage 2, the resulting representation is compared serially to each letter of the memory set. In Stages 3 and 4 , S makes a decision and responds according to the results of Stage 2 . In terms of the reaction-time functions, the intercept of a function includes the sum of the times devoted to the first, third, and fourth stages, whereas the slope of the function for negative responses represents the time required during Stage 2 for each comparison of a memory-set element with the test stimulus. If the comparison process is terminated and a response is made immediately after the test stimulus is matched with a memory-set element (a self-terminating scan), the ratio of the slope of the function for negative responses to the slope for positives should be two to one (since, for a positive trial, the test stimulus is matched on the average after half the memory set has been scanned). If $S$ initiates a response only after all memory-set elements have been compared to the test stimulus, regardless of whether or not a match is made (an exhaustive scan), the slope of the functions for positive and negative responses should be equal.

In one variant of the memory scanning task, the test stimulus is a picture of a familiar object rather than a letter. In this case, $S$ makes a positive response only if the first letter of the name of that object is a member of the memory set (e.g., $T$ for a picture of a tree). This variant of the scanning task requires that verbal processing (naming the picture) precede the response.

In previous experiments (Klatzky \& Atkinson, 1970; Klatzky, Juola, \& Atkinson, 1971) with the memory scanning paradigm described above, it was found that the slopes of the reaction-time functions for picture test stimuli were approximately twice the slopes of the functions for letter test stimuli. Assuming that the slope of the function represents the time required to make a single comparison during Stage 2, the slope difference suggests that the test stimulus representations used in the scanning process differed in the two cases. Since the transformation of a picture test stimulus to the initial letter of its name is a verbal process, it is possible that the resulting test stimulus representation is a verbal label of that letter (e.g., "ess" when the test stimulus is a picture of a snake). In contrast, the representation of a letter test stimulus may be a spatial pattern, as Stemberg (1969) has suggested. Thus, the differences in the slopes of the reaction-time functions for the two types of stimuli led to the hypothesis that letters are spatially represented and pictures are verbally represented in the scanning process.

In view of theories of hemispheric specialization (Levy, 1969; Levy-Agresti \& Sperry, 1968; Sperry \& Levy, 1970), the above hypothesis implies that the comparison process might be performed by the left hemisphere when the test stimulus is a picture and by the right hemisphere when it is a letter. As a result, the presentation of a letter test stimulus to the left hemisphere requires its transfer to the right before spatial comparisons can be made. The transfer causes an increase in the time devoted to Stage 1, which raises the intercept of the reaction-time function for left-hemisphere presentations of letter test stimuli relative to the function for the right hemisphere. Similarly, a picture test stimulus presented to the right hemisphere must be transferred to the left for verbal processing, raising the intercept of the function for right-hemisphere presentations relative to the function for presentations to the left hemisphere. Assuming that the test stimulus representation used for comparisons is unaffected by interhemispheric transfer, the slopes of 
the functions for left- and right-hemisphere presentations should be identical.

These predictions were tested in a preliminary experiment (Klatzky, 1970), and the results appeared to support the hypothesis that letter and picture test stimuli are represented as spatial forms and verbal labels, respectively, and the two types of stimuli are processed by different cerebral hemispheres. In that experiment the test stimulus presented on a given trial could be either a letter or a picture, with a $50 \%$ probability of each type. However, an earlier study (Klatzky et al, 1971) has shown that a mixed presentation of pictures and letters results in processing that is markedly different from that found when a test stimulus type remains constant over a long series of trials. For this reason, it was decided to expand on the preliminary experiment, using the condition of nonmixed stimulus presentation. In the present experiment, therefore, two types of sessions were used: letter sessions, in which the test stimulus on every trial was a letter, and picture sessions, in which each test stimulus was a picture.

\section{Subjects}

The Ss were eight right-handed female students at Stan ford University who spoke English as their native language. They were paid $\$ 2.00$ for each of nine experimental sessions.

\section{Stimuli}

The memory-set stimuli consisted of 72 slides prepared from photographs of letters typed with an IBM Executive Registry electric typewriter. The number of letters in a memory set varied from two to five. A dollar sign (\$) was placed at each end of the display to delimit it; there were no spaces between the ends of the display and their delimiters. The set of letters used in memory-set displays consisted of all members of the alphabet except the five vowels and $V, X$, and $Y$; this set of letters will be referred to as the letter set. Each member of the letter set was used equally often in each serial position in memory sets, and no letter was ever duplicated within a memory set.

There were two types of test stimuli: letters and pictures. Letter-test stimuli were displayed on slides which were prepared in the same manner as memory-set displays. However, no dollar signs were present on letter slides. There was a letter stimulus corresponding to each member of the letter set. Also corresponding to each letter-set element were three picture-test stimuli. All three stimuli represented a single common noun whose first letter was the member of the letter set (e.g., there were three different pictures of dogs used as stimuli, each representing the letter $D$, three different snake pictures for the letter S, etc.). These pictures were presented on slides prepared from photographs of black-and-white drawings.

\section{Apparatus}

The apparatus consisted of an Iconix automated tachistoscope and exposure box. The controls of the apparatus were located in an adjacent room, where a punched paper tape read by a Teletype controlled the sequence of trial events. The data of each trial were punched automatically onto a paper tape and also printed by a Teletype.

The stimuli were presented to $S$ through a circular aperture onto a viewing screen, illuminating a circle with a diameter of $2-1 / 16$ in. The projections of memory-set displays were $3 / 16 \mathrm{in}$. high, and their width varied from $1 / 2 \mathrm{in}$. for a display of Size 2 to $7 / 8 \mathrm{in}$. for a display of Size 5. The picture slides, when projected, had both a maximum height and a maximum width of $2-1 / 16$ in., the diameter of the viewing circle. The line-of-sight viewing distance was approximately $2 \mathrm{ft}$.

Above the viewing aperture, three small colored lights could be illuminated. These were used to indicate to $S$ whether or not a response was correct. Below the viewing screen was an IEE Binaview unit which was used to signal $S$ to begin the test portion of the trial. Two small dots, located 2 in. to the right and left of the center of the viewing area, were used as fixation points.

On a table in front of $S$ were three telegraph keys arranged in an arc, with their centers separated by a distance of $1.25 \mathrm{in}$. The $\mathrm{S}$ rested one arm on the table and depressed the keys with her forefinger. Four Ss were chosen at random to press the key on the right for a positive response and the key on the left for a negative response, and the remaining $\mathrm{Ss}$ used the reverse procedure. Each $S$ was instructed to depress the center key until she was ready to respond, so that her hand position was not biased in favor of either of the response keys. A start button held in the nonresponse hand was used by $S$ to terminate the memory set and to initiate the test stimulus.

\section{Procedure}

Each $S$ participated in one training session and eight additional test sessions of 160 trials each; each session lasted about $1 \mathrm{~h}$. Two types of test sessions were used: letter sessions, in which only letters were used as test stimuli, and picture sessions, in which only pictures appeared on tests. The first, or training, session consisted of two sequences of trials: one using only letters as test stimuli, the other using only pictures. The $S$ subsequently participated in four letter sessions alternating with four picture sessions.

The hemisphere to which the test stimulus was presented was determined by the direction of fixation during the test. If $S$ was instructed to fixate upon the dot to the left of the viewing area, the test stimulus appeared in the right visual field and was projected to the left hemisphere, with the situation reversed for right fixation. The direction of fixation alternated every 40 trials within an experimental session, according to E's instructions.

The hand used for responses was also varied within each session. The $S$ used one hand for responses during the first half of the session and then used the other hand during the second half.

The order of test-session types, direction of fixation during the first 40 trials, and response hand used during the first half of a session were arranged according to a Latin-square design. Each memory-set size (two, three, four, or five), each response (positive or negative), and each serial position for a correct response (the position of the test stimulus in the memory set, numbered from left to right) was chosen randomly with equal probability for use in a given session; and on the average, each was used equally often in a session.

At the beginning of each picture session, copies of the pictures which were to be used as test stimuli were viewed and named by $S$ before starting the series of 160 trials. Each trial lasted approximately $16 \mathrm{sec}$ and involved the following sequence of events: (1) A memory set, delimited by dollar signs, appeared on the screen in front of S. (2) The S looked directly at the set until she had memorized it and then turned it off by pushing the start button. (3) About 5 sec later, the Binaview unit flashed and an auditory signal of two clicks indicated to $S$ to begin the test as soon as she was ready. (4) The $S$ then fixated on the left or right fixation point, according to E's instructions, and again pressed the start button. After 500 msec, the test stimulus appeared on the screen for 400 msec. $^{1}$ (5) Using her response hand, $S$ lifted her forefinger from the center key and depressed the key to the right or left. (6) A red, green, or white light then appeared on the screen, indicating whether $\mathbf{S}$ had made an incorrect response, had made a correct response, or had exceeded a 


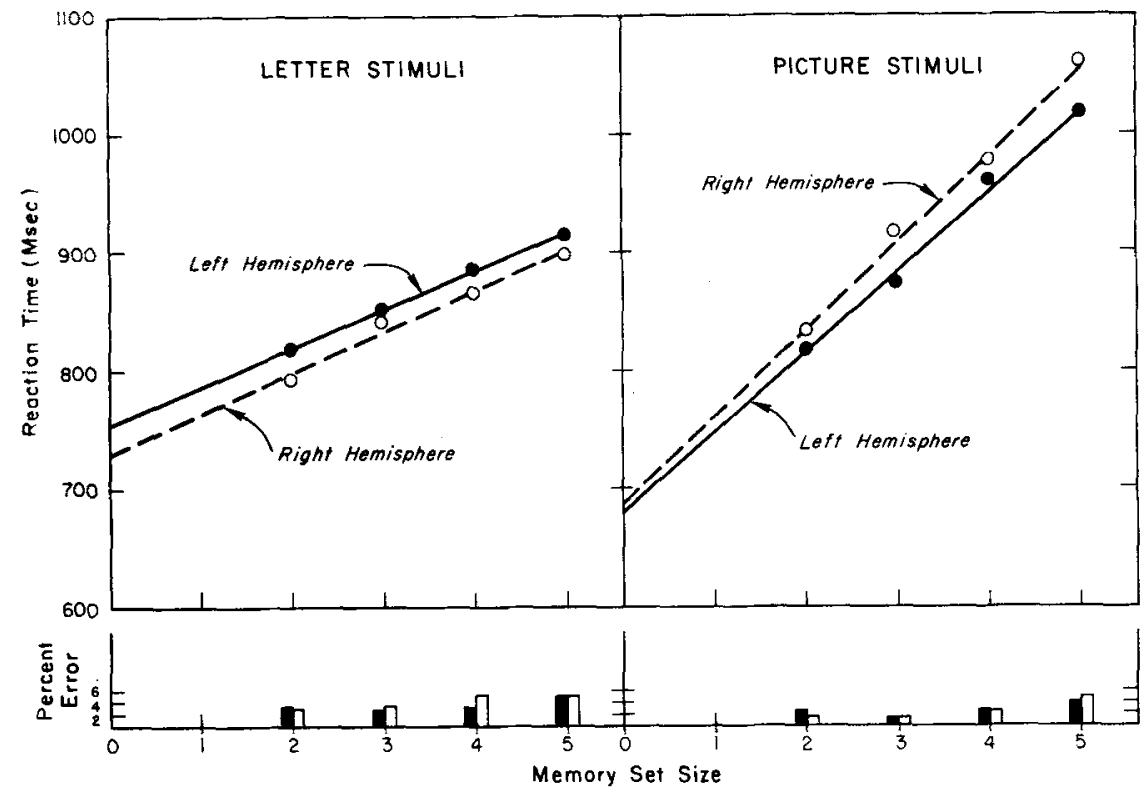

Fig. 1. Reaction time in milliseconds and percent errors for responses to letter (left panel) and picture (right panel) test stimuli as a function of memory-set size (averaged over Ss, positive and negative responses, and right and left responding hands). Right-hemisphere presentations are indicated by white circles and bars; left-hemisphere presentations are indicated by black circles and bars. The linear functions, calculated by the method of least squares, are as follows: for letters, $R T(d)=753.6+32.0 d$ for left-hemisphere presentation and $R T(d)=$ $728.4+34.8 d$ for right-hemisphere presentation; for pictures, $R T(d)=678.8+$ 68.4d for left-hemisphere presentation and $R T(d)=683.5+74.9 \mathrm{~d}$ for right-hemisphere presentation, where $R T(d)$ is the reaction time associated with a memory set of Size d.

2-sec limit on the time allotted between the test stimulus onset and her response, respectively. (7) After an interval of $5 \mathrm{sec}$, the next trial began.

The S's response time was recorded by a latency counter, the onset of which was simultaneous with the onset of the test stimulus. The counter was terminated when the key to the right or left was depressed.

After Trials 40,80 , and $120, \mathrm{E}$ entered S's room to change slide drums for the projector and to instruct $S$ which hand and fixation point to use for the next 40 trials. This procedure provided a rest period for $\mathrm{S}$ lasting about $2 \mathrm{~min}$.

\section{RESULTS AND DISCUSSION}

The principle results are mean reaction times (RT) in milliseconds; RT is defined as the time between the onset of the test stimulus and S's response. The data analysis includes the data for correct responses only; however, the error rates were low. Over individual Ss, the error rates had a range of $1.4 \%$ to $4.8 \%$, with a mean of $3.4 \%$. In addition, the first six trials of each test session were considered warm-up trials, and the data from these trials were not included in the analysis. Data from the initial training sessions were also excluded.
Figure 1 presents the RT functions for each type of session and each hemisphere of test stimulus presentation, showing RT as a function of the size of the memory set. The error rates corresponding to each point in the functions are also indicated. The mean RT, averaged over Ss and memory-set sizes, for each test stimulus type, hemisphere of test stimulus presentation, responding hand, and response type (positive or negative) is given in Table 1 .

The reaction-time functions for leftand right-hemisphere presentations of letter and picture test stimuli shown in
Fig. 1 are linear and increasing. When these data are broken down into positive and negative responses and right- and left-hand responding, the functions are again linear and increasing. Moreover, within each test stimulus type (letters or pictures), the functions for both responses have approximately equal slopes. These results conform to the predictions of Sternberg's (1966) model for exhaustive memory scanning. However, the serial position functions (which relate reaction time for positive responses to the position of the test stimulus in the memory set) are all increasing. This result, which was also obtained in our previous research with this paradigm (Klatzky \& Atkinson, 1970; Klatzky et al, 1971; Klatzky, 1970), is not predicted by the exhaustive scanning model unless additional assumptions are made.

Examination of the data reveals that the slopes of the reaction-time functions for letter stimuli are approximately half the slopes for pictures, as expected on the basis of previous research (Klatzky \& Atkinson, 1970; Klatzky et al, 1971). This supports the hypothesis that test stimulus representations of letters and the corresponding pictures are not identical. Moreover, the hypothesis that the representations are spatial and verbal-acoustic for letter and picture stimuli, respectively, also receives support (Fig. 1). The reaction-time function for left-hemisphere presentation of letters is approximately parallel to and above the function for right-hemisphere presentation, and this arrangement is reversed for the picture test stimuli. This interaction between hemisphere of presentation and test stimulus type is independent of response type (positive or negative) and responding hand (right or left). Moreover, a three-way analysis of variance performed over $S s$, hemisphere of presentation, and test stimulus type indicates that this Hemisphere by Stimulus Type interaction is

Table 1

Reaction Time in Milliseconds (Averaged Over Ss and Memory Set Sizes) for Each Test Stimulus Type, Hemisphere of Test Stimulus Presentation, Responding Hand, and Response Type

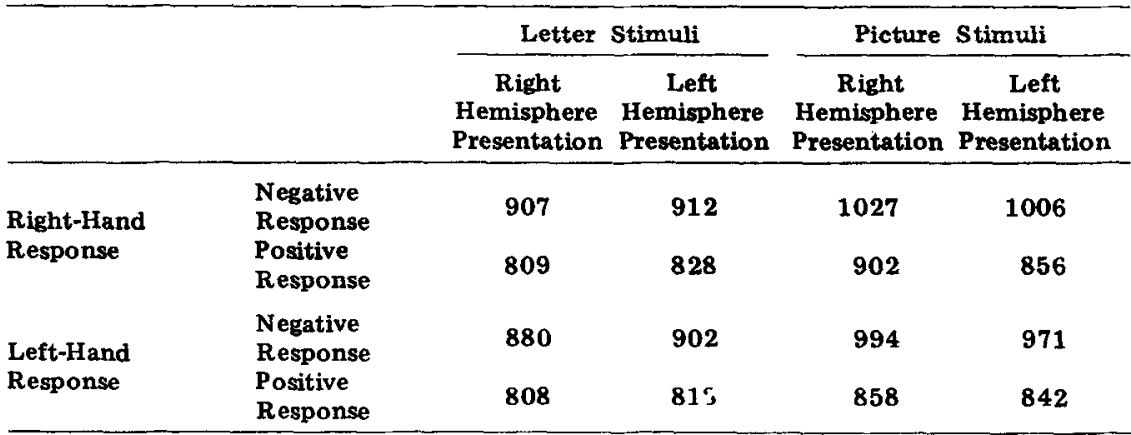


significant $[F(1,7)=5.87 ; p<.05]$. Presumably, the difference in intercepts for right- vs left-hemisphere presentation corresponds to the time associated with the transfer of an incoming test stimulus to the hemisphere responsible for its processing-the right hemisphere for letter stimuli and the left for pictures. That the slopes of the functions are the same for presentation to both hemispheres implies that if a test stimulus is transferred, processing proceeds as if it had initially entered the appropriate hemisphere.

It had previously been found (Klatzky \& Atkinson, 1970; Klatzky et al, 1971) that the intercepts of pieture functions were greater than letter function intercepts, a difference assumed to correspond to the greater amount of Stage 1 processing (i.e, naming the picture and extracting the first letter of that name) which must occur before representations of picture test stimuli can be compared to the memory set. In this experiment, the intercepts of letter functions are slightly greater than intercepts of picture functions. However, the relative increase in the letter function intercepts may be attributed to difficulty in initially perceiving the letter stimuli when presentation is peripheral; Ss reported that picture stimuli, which are larger than the letters, were easier to identify.

The reaction-time functions for right- vs left-hand responses have approximately equal slopes and show a lower intercept for left-hand responses, a difference which is larger for picture test stimuli than for letters (43.1 vs $26.0 \mathrm{msec}$ ). Since the response keys are mounted on one corner of a rectangular board which was rotated $90 \mathrm{deg}$ when $S$ changed her responding hand, it is possible that a left-hand advantage resulting from the asymmetry of the response panel may exist. If this is true, the data indicate that such an advantage must be enhanced for picture stimuli and diminished in the case of letters. Since other Es have found that reaction time is faster when a response controlled by one hemisphere is performed by the contralateral rather than the ipsilateral hand (Bradshaw \& Perriment, 1970; Berlucchi, Heron, Hyman, Rizzolatti, \& Umilta, in press), these functions suggest that responses to letter and picture stimuli are initiated by the left and right hemisphere, respectively. Thus, it appears that the hemisphere which initiates the response is not the same as the hemisphere which performs the comparisons during the second stage of the scanning process. We suggested in a recent paper (Klatzky et al, 1971) that the comparison (Stage 2) and decision (Stage 3) components of memory scanning are somewhat independent; this experiment indicates that the two components are controlled by different hemispheres. Possibly, limitations in the processing capacity of the comparison-performing hemisphere require the other hemisphere to monitor the comparisons and initiate the response. If the hand contralateral to the responding hemisphere is not used for responses, the response information must be transferred to the opposite hemisphere, increasing the time devoted to the third scanning stage, and, in turn, raising the intercept of the reaction-time function.

Emerging from the data of this experiment is a picture of the cerebral hemispheres as two informationprocessing systems which optimize performance by specializing in different functions and, when capacity is limited, sharing the processing load. Research with brain-bisected patients (Sperry \& Levy, 1970) suggests that either the left or right hemisphere could perform a memory scanning task with letter stimuli on the basis of verbal or spatial stimulus representations, respectively. Spatial comparisons of letters are undoubtedly faster than a verbal-acoustic comparison process; consequently, right-hemisphere processing of letters appears to be favored. In contrast, the right hemisphere seems to lack the verbal capacity required to transform the picture of an object to the initial letter of its name. Because pictures must be converted to letters by complex verbal manipulation, comparisons based on picture stimuli appear to be left-hemisphere functions, and the test stimulus representations are probably verbal labels. While the processing capacity of one hemisphere is occupied by comparisons, the response is supervised by the other. The operation of this dual system is revealed by response latency increments which, in theory, correspond to communication between two separate but cooperating information processors.

\section{REFERENCES}

BERLUCCHI, G., HERON, W., HYMAN, R., RIZZOLATTI, G., \& UMILTA.C. Simple reaction times of ipsilateral and contralateral hand to lateralized visual stimuli. Brain, in press.

BRADSHAW, J. L., \& PERRIMENT, A. D. Laterality effects and choice reaction time in a unimanual two-finger task. Perception \& Psychophysics, 1970, 7, 185-188.

FILBEY, R. A., GAZZANIGA, M. S. Splitting the normal brain with reaction time. Psychonomic Seience, 1969, 17, 335-336.

KLATZKY, R. L. Interhemispheric transfer of test stimulus representations in memory scanning. Psychonomic Science, 1970, 21, 201-203.

KLATZKY, R. L.. \& ATKINSON, R. C. Memory scans based on alternative test stimulus representations. Perception \& Psychophysics, 1970, 8, 113-117.

KLATZKY, R. L., JUOLA, J. F., \& ATKINSON, R. C. Test stimulus representation and experimental context effects in memory scanning. Journal of Experimental Psychology, 1971. 87, 281-288.

LEVY, J. Possible basis for the evolution of lateral specialization of the human brain. Nature, 1969, 224, 614-615.

LEVY-AGRESTI, J, \& SPERRY, R. W . Differential perceptual capacities in major and minor hemispheres. Proceedings of the National Academy of Sciences, 1968 , 61,1151 .

SPERRY, R. W., \& LEVY, J. Mental capacities of the disconnected minor hemisphere following commissurotomy. Paper presented at the symposium on asymmetrical function of the human brain, annual convention of the American Psychological Association, Miami, Florida, September 1970.

STERNBERG, S. High-speed scanning in human memory. Science, 1966, 153, $652-654$.

STERNBERG, S. Memory-scanning: Mental processes revealed by reaction-time experiments. American Scientist, 1969. $57,421.457$.

\section{NOTE}

1. Although a 400-msec exposure time does not prohibit $S$ from making eye movements which could expose the test stimulus to both hemispheres, pretesting indicated that at shorter intervals, $S$ could not identify the test stimulus on a large number of trials. All Ss were instructed to keep their eyes on the fixation point throughout test stimulus presentation, and they reported that these instructions were followed. Since exposure of the test stimulus to both hemispheres would eliminate the need for interhemispheric transfer, the effect of eye movements would be to reduce or eliminate differences in $R T$ associated with different hemispheres of presentation.

(Accepted for publication May 12, 1971.) 